Z Rheumatol 2007 · 66:7-7

DOI 10.1007/s00393-007-0147-7

(c) Springer Medizin Verlag 2007
A. Radbruch

Deutsches Rheuma-Forschungszentrum Berlin (DRFZ)

\title{
Lebendige Rheumatologie
}

Die Rheumatologie hat in Deutschland in den letzten Jahren eine beeindruckende Entwicklung genommen, eine Entwicklung, die sich in ihrer wissenschaftlichen Fachgesellschaft spiegelt, der Deutschen Gesellschaft für Rheumatologie (DGRh). Dies ist nicht nur, aber ganz wesentlich einer starken Präsidentin, Elisabeth Märker-Hermann (2005/2006) und starken Präsidenten zu verdanken, ihrer Energie, Weitsicht und Überzeugungskraft - auch ihrem Charme! Es ist eine Ehre und eine Verpflichtung, diesen Vorbildern nachzufolgen. Durch die Einrichtung einer leistungsfähigen $\mathrm{Ge}$ schäftsstelle mit den Geschäftsführern Cornelia Rufenach und Andreas Heinrich ist die Arbeit für die Gesellschaft einfacher geworden und Neues wird möglich. Die neugeschaffene Position eines Generalsekretärs hat sich bewährt. Erika Gromnica-Ihle hat diese Position eindrucksvoll ausgefüllt und ihr Format gegeben. Seit Anfang des Jahres 2007 ist Ekkehard Genth Generalsekretär, ein bewährter Kämpe und Kenner der Gesellschaft. Ich freue mich auf eine enge und konstruktive Zusammenarbeit. Es gibt viel zu tun.

Vieles ist bereits erreicht worden: Die Einbindung des medizinischen Kompetenznetzwerkes „Entzündlich-rheumatische Erkrankungen" hat die Forschung eng in die Gesellschaft integriert.

\section{- Die Stärkung der wissenschaftlichen Aktivitäten in der DGRh ist wichtiger Teil der Fachentwicklung.}

Die Rheumaakademie wurde gegründet als neue Plattform für Weiterbildung, Fortbildung und wissenschaftliche Kommunikation. Die Partnerschaft mit der pharmazeutischen Industrie ist von gegenseitigem Respekt geprägt. Die europäische Dimension wurde gestärkt durch den EULAR-Kongress in Berlin, mehr noch über die beeindru- ckende Vernetzung der rheumatologischen Forschung über den Europäischen Workshop für Rheumaforschung „EWRR“, die EULAR/EU-Arbeitsgemeinschaft „CellAid“ und das europäische Forschungskonsortium „Autocure“. Deutsche Rheumatologen sind gern gesehene und respektierte Partner. An der Erarbeitung des politischen Positionspapiers der Europäischen Wissenschaftsorganisation ESF zur rheumatologischen Forschung waren deutsche Rheumatologen federführend beteiligt.

\section{( Mit der neuen Rubrik "Neues aus der Forschung" immer up-to-date}

Auf nationaler Ebene sind die rheumatologischen Jahrestagungen Höhepunkte im Leben der Gesellschaft. Sie haben sich zu einer breiten, integrativen Kommunikationsplattform entwickelt, von der Grundlagenforschung bis zur Patientenschulung. Der Dialog zwischen den verschiedenen Expertengruppen, Ärzten und Patienten bleibt eine Herausforderung. Es ist schwer, der rasanten Entwicklung der rheumatologischen Forschung zu folgen und aus der großen Zahl der wissenschaftlichen Veröffentlichungen die "Rosinen" herauszupicken. Wir haben deshalb in der Zeitschrift für Rheumatologie eine neue Rubrik „Neues aus der Forschung " eingerichtet, in der Wissenschaftler der Gesellschaft aus ihrer Sicht interessante Entwicklungen darstellen.

Beginnen wird Prof. Thomas Kamradt aus Jena mit einem Bericht über Th17-Lymphozyten. Seine Arbeitsgruppe hatte diese Zellen erstmals in entzündeten Gelenken von Patienten mit Rheumatoider Arthritis gefunden, heute gelten sie als eine treibende Kraft chronischer Entzündungen. Viel Spaß beim Lesen!

Forschung ist teuer. Die Deutsche Gesellschaft für Rheumatologie hat bereits be- trächtliche Eigenmittel zur Verfügung gestellt, um eine erste Runde des „Start-Up“Förderprogramms zu ermöglichen. Durch die Gründung der „Stiftung Rheuma“, gemeinsam mit der Rheumaliga, wollen wir in Deutschland das Bewusstsein für das Potential rheumatologischer Forschung wecken und Spendengelder einwerben, um sie zu finanzieren.

Forschung braucht auch klare Ziele und exzellente Forscher. Die Exzellenz wird durch die gekonnte Einbindung der modernen Biologie und die Rekrutierung junger Mediziner in die Rheumatologie erreicht. Die Ziele werden durch den Dialog mit Ärzten und Patienten definiert. Als interdisziplinäre Wissenschaft integriert die Rheumatologie dabei Entzündungsforschung, Osteologie, Rheumaorthopädie, regenerative Rheumatologie, verschiedene Bereiche der Diagnostik, der Therapie- und Schmerzforschung, sowie andere Felder der Versorgungsforschung, um nur einige zu nennen. Die Rheumatologie ist zugleich umfassende Wissenschaft und attraktives Berufsbild: Rheumatologie macht Spaß!
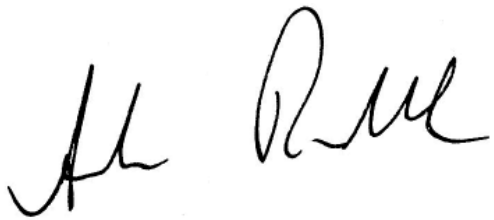

Andreas Radbruch

Präsident

Deutsche Gesellschaft für Rheumatologie

\section{Korrespondierender Autor}

\section{Prof. Dr. A. Radbruch}

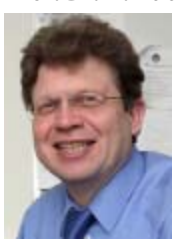

Deutsches Rheuma-

Forschungszentrum

Berlin (DRFZ)

Charitéplatz 1, 10117 Berlin

radbruch@drfz.de 